\title{
Comportamento Sedentário em Adolescentes: Análise Hierárquica de Fatores Associados
}

\author{
Thiago Silva Piola ${ }^{1}$ Eliane Denise Araújo Bacil ${ }^{2}$, Michael Pereira Silva ${ }^{3}$, \\ Jhonatan Gritten Campos ${ }^{4}$, Nicolau Augusto Malta Neto ${ }^{5}$, Wagner de Campos ${ }^{6}$
}

\begin{abstract}
RESUMO
Objetivo: verificar os fatores associados ao tempo de tela, tempo em atividades educacionais, culturais, sociais e de transporte em adolescentes. Métodos: estudo correlacional de corte transversal, com amostra representativa de adolescentes de São José dos Pinhais-PR $(n=785)$. Os comportamentos sedentários autorreportados foram o tempo em atividades de tela, educacionais, culturais, sociais e transporte. Os fatores associados ao comportamento sedentário foram o sexo, nível de atividade física, nível socioeconômico, maturação sexual e estado nutricional. As associações foram testadas com a regressão de Poisson. Resultados: o sexo feminino foi associado ao elevado tempo de tela (RP: 0,83; IC95\%: 0,72- 0,96), em atividades educacionais (RP: 1,62; IC95\%: 1,38-1,90), em atividades culturais (RP: 0,41; IC9\%: 0,30-0,56) e sociais (RP: 0,58; IC95\%: 0,53$0,64)$. O nível insuficiente de atividades físicas se associou às atividades culturais (RP: 0,68 ; IC95\%: $0,49-0,94$ ) e ao tempo em transporte (RP: 0,51; IC95\%: 0,45-0,58). A maturação sexual foi associada às atividades sociais (RP: 0,80; IC95\%: 0,68-0,95). O estado nutricional associou-se às atividades culturais (RP: 1,62; IC95\%: 1,04-2,52). Conclusões: os achados sugerem que diferentes tipos de comportamento sedentário podem estar associados ao sexo feminino, nível de atividade física, maturação sexual e estado nutricional, e esses fatores devem ser considerados em futuras intervenções para a promoção da saúde.
\end{abstract}

Palavras-chave: Comportamento sedentário. Atividade motora. Classe social. Estado nutricional.

\section{SEDENTARY BEHAVIOR IN ADOLESCENTS: HIERARCHICAL ANALYSIS OF ASSOCIATED FACTORS}

\section{ABSTRACT}

Objective: to verify associated factors of screen time, time spent in educational, cultural and social activities and transport in adolescents. Methods: cross-sectional correlational study with a representative sample of adolescents from São José dos Pinhais-PR $(n=785)$. The sedentary behaviors, self-supported, were screen time, educational, cultural, social and transportation activities. The measures associated with behavior were: sex, physical activity level, socioeconomic level, sexual maturation and nutritional status. Poisson regression verified de associations. Results: Female gender was associated with high screen time (PR: 0.83; CI95\%: $0.72-0.96)$, educational time (PR: 1.62; CI95\%: 1.38-1.90), cultural activities (PR: 0.41; Cl95\%: $0.30-0.56$ ) and social (PR: 0.58; Cl95\%: $0.53-0.64)$. Insufficient physical activity level is associated to cultural activities (PR: 0.68; CI95\%: $0.49-0.94$ ) and transport time (PR: 0.51; Cl95\%: $0.45-0.58$ ). Sexual maturation was associated with social activities (PR: 0.80; CI95\%: $0.68-0.95$ ). Nutritional status was associated with cultural activities (PR: 1.62; CI95\%: $1.04-2.52$ ). Conclusions: different sedentary behaviors can be explained about female sex, physical activity level, sexual maturation and nutritional status. Health promote interventions need include these factors.

Keywords: Sedentary behavior. Motor activity. Social class. Nutritional status.

RECEBIDO EM: 5/8/2019

MODIFICAÇÕES REQUERIDAS EM: 6/9/2019

ACEITO EM: 12/9/2019

\footnotetext{
Doutor em Educação Física pela Universidade Federal do Paraná (UFPR). Membro do Centro de Estudos em Atividade Física e Saúde (CEAFS/UFPR). Professor do Centro Universitário Claretiano. Possui experiência na área de Atividade Física e Saúde com ênfase na saúde da criança e do adolescente. tspthiago@hotmail.com Doutora em Educação Física pela Universidade Federal do Paraná (UFPR). Membro do Centro de Estudos em Atividade Física e Saúde (CEAFS/UFPR). Professora da Universidade Positivo e professora substituta da Universidade Federal do Paraná nos cursos de Licenciatura e Bacharelado em Educação Física. Tem experiência na área de Educação Física, com ênfase em Atividade Física e Saúde, Epidemiologia da Atividade Física, Crescimento e Desenvolvimento e Medidas e Avaliação. elianebacil@hotmail.com

${ }^{3}$ Doutor em Educação Física pela Universidade Federal do Paraná (UFPR). Membro do Centro de Estudos em Atividade Física e Saúde (CEAFS/UFPR). Professor da Universidade Estadual do Centro-Oeste. Possui experiência na área de Atividade Física e Saúde com ênfase na saúde da criança e do adolescente. prof.mpsilva@outlook.com

${ }^{4}$ Doutorando em Educação Física pela Universidade Federal do Paraná (UFPR). Membro do Centro de Estudos em Atividade Física e Saúde (CEAFS/UFPR). jhonatantec@hotmail.com

${ }^{5}$ Mestrando em Educação Física pela Universidade Federal do Paraná (UFPR). Membro do grupo de pesquisa Centro de Estudos em Atividade Física e Saúde (CEAFS/UFPR). nicolaumalta@hotmail.com

${ }^{6}$ Doutorado em Desenvolvimento Motor e Estudos dos Esportes pela University of Pittsburgh, PITT, Estados Unidos. Líder do Centro de Estudos em Atividade Física e Saúde (CEAFS/UFPR). Professor titular na Universidade Federal do Paraná (UFPR). wagner-campos@hotmail.com
} 


\section{INTRODUÇÃO}

A literatura científica apresenta resultados alarmantes sobre o pouco engajamento de adolescentes em atividade física (HALLAL et al., 2012; BARBOSA FILHO; CAMPOS; LOPES, 2014). Por outro lado, estes baixos níveis de atividade física parecem estar associados ao elevado tempo em comportamento sedentário (GUERRA; FARIAS JÚNIOR; FLORINDO, 2016). Este, por sua vez, parece ocupar grande parte do dia dos adolescentes, quando estudos mostram prevalências acima de $50 \%$ de elevado comportamento sedentário por adolescentes brasileiros (BARBOSA FILHO; CAMPOS; LOPES, 2014).

A exemplo da prática de atividades físicas (BAUMAN et al., 2012), o comportamento sedentário também se associa a diferentes fatores (GUERRA; FARIAS JÚNIOR; FLORINDO, 2016), como o sexo (TENÓRIO et al., 2010), o nível de atividade física (DIAS et al., 2014), o nível socioeconômico (DIAS et al., 2014), a maturação sexual (BACIL et al., 2016) e o estado nutricional (DIAS et al., 2014), entretanto, para a maioria destes fatores, a literatura ainda apresenta resultados conflitantes e inconclusivos acerca destas associações (BARBOSA FILHO; CAMPOS; LOPES, 2014; GUERRA; FARIAS JÚNIOR; FLORINDO, 2016; KANTOMAA et al., 2016).

Se, por um lado, fatores possivelmente associados ao comportamento sedentário estão sendo investigados pela literatura (BARBOSA FILHO et al., 2012; FERREIRA et al., 2016; GUERRA; FARIAS JÚNIOR; FLORINDO, 2016), os estudos disponíveis tendem a considerar como desfecho principal basicamente o tempo de tela (BARBOSA FILHO et al., 2012; BARBOSA FILHO; CAMPOS; LOPES, 2014; FERREIRA et al., 2016; GUERRA; FARIAS JÚNIOR; FLORINDO, 2016), o que pode ser compreendido, pois um elevado tempo de tela está associado significativamente a maiores taxas de mortalidade, mesmo em sujeitos com adequados níveis habituais de atividades físicas (EKELUND et al., 2016).

Comportamento sedentário, no entanto, não pode ser definido em sua totalidade apenas por atividades defronte à tela. Em sua definição clássica, este caracteriza-se por atividades de baixa movimentação, baixo gasto energético (< 1,5 MET) e que ocorrem basicamente na posição sentada ou reclinada (AINSWORTH et al., 2000; PATE; O'NEILL; LOBELO, 2008), ou seja, contemplam mais atividades do que apenas o tempo de tela, podendo englobar atividades cotidianas, como falar ao telefone, conversar com amigos, hobbies, como tocar instrumentos musicais ou jogar cartas, dirigir, se deslocar sentado em transporte coletivo, tempo sentado na escola ou no trabalho, inclusive assistir televisão, usar o computador e jogar video game (HARDY; BOOTH; OKELY, 2007; GUERRA; FARIAS JÚNIOR; FLORINDO, 2016).

Partindo deste pressuposto, o presente estudo teve por objetivo: i) verificar as prevalências do elevado tempo sedentário em atividades de tempo de tela, atividades educacionais, culturais e sociais, além do tempo sedentário relacionado ao deslocamento; e ii) verificar as possíveis associações do sexo, do nível de atividade física, do nível socioeconômico, da maturação sexual e do estado nutricional com o elevado tempo de tela, atividades educacionais, culturais e sociais, além do tempo sedentário relacionado ao deslocamento.

\section{MÉTODOS}

\section{Design}

Trata-se de um estudo correlacional de corte transversal, com uma amostra representativa de adolescentes ( $n=10523$ ) matriculados no Ensino Médio das escolas estaduais do município de São José dos Pinhais, Paraná, Brasil (SEED-PR, 2014). O município citado é parte da grande Curitiba, sendo o $5^{\circ}$ maior em extensão no Estado do Paraná. Seu índice de desenvolvimento humano é considerado alto $(0,758)$, ocupando a 400a posição em relação aos 5.565 municípios do Brasil (BRASIL, 2016).

\section{Amostra}

O cálculo amostral, a priori, para o estudo, foi realizado em duas etapas. Primeiramente foi estimado o $n$ mínimo necessário para calcular as prevalências dos desfechos de interesse a partir dos procedimentos sugeridos por Luiz e Magnanini (2000), considerando um erro amostral de $5 \%$, uma prevalência do elevado tempo sedentário em 50\% (BARBOSA FILHO; CAMPOS; LOPES, 2014) (o que também garante um $n$ máximo ao cálculo), estimando um $n$ mínimo de 371 adolescentes para o estudo. Ponderando um efeito de delineamento de 1,5 e um acréscimo de $30 \%$, prevendo possíveis perdas e recusas, a amostra mínima para estimar as prevalências do desfecho foi de 723 sujeitos.

A segunda etapa do cálculo amostral, a priori, foi realizada para estimar a amostra mínima necessária para testar a hipótese do estudo (DEMIDENKO, 2007), considerando uma associação de 0,73 entre o sexo e o excessivo tempo de tela, com uma prevalência de 64,5\% (TENÓRIO et al., 2010), um nível de confiança de $95 \%(\alpha=0,05)$ e um poder de $80 \%(\beta=$ 20), resultando em uma amostra mínima de 574 sujei- 
tos, com uma probabilidade de rejeitar corretamente a hipótese nula de $80 \%$. Com um acréscimo de $30 \%$ para prevenir as possíveis perdas e recusas, a amostra mínima para o teste de hipótese foi estabelecida em 746 sujeitos.

Para a coleta de dados, três estágios foram realizados, sendo: i) a seleção de todas as escolas do município que ofertassem o Ensino Médio matutino; ii) a seleção aleatória simples, de uma turma de cada série do Ensino Médio ( $1^{\circ}, 2^{\circ}$ e $3^{\circ}$ anos); e iii) o convite de todos os alunos da turma sorteada para participar do estudo. A coleta de dados foi realizada em sala de aula por avaliadores previamente treinados do Centro de Estudos em Atividade Física - Ceafs/UFPR. $O$ estudo seguiu as normas de pesquisa envolvendo seres humanos estabelecidas pelo Conselho Nacional de Saúde (resolução $n^{\circ}$ 466/2012) e foi aprovado pelo Comitê de Ética em Pesquisa da Universidade Federal do Paraná (CAAE: 36759414.0.0000.0102).

Foram avaliados 942 adolescentes entre abril e junho de 2014. Foram excluídos das análises, porém, aqueles que apresentaram limitações físicas $(n=2)$ e os que reportaram estágio maturacional pré-púbere $(n=26)$. Também foram excluídos os adolescentes que não entregaram o Termo de Consentimento Livre e Esclarecido assinado pelos pais ou responsáveis, os que se negaram a participar do estudo, os que preencheram os instrumentos de maneira incorreta e/ou incompleta ou ainda faltaram no dia da coleta de dados $(n=130)$.

Com isso, a amostra final do estudo contemplou 785 adolescentes entre 15 e 17,9 anos (classificados como adolescentes de acordo com os critérios da Organização Mundial da Saúde (WHO, 2017).

Para verificar o poder estatístico da amostra foi realizado um cálculo a posteriori, considerando os mesmos parâmetros do teste de hipótese $a$ priori $(\alpha=$ 0,05 e $\beta=0,20$ ) e as prevalências para cada desfecho do comportamento sedentário observados no presente estudo.

\section{Instrumentos e Procedimentos}

O sexo foi autorreportado pelos adolescentes e categorizado em masculino e feminino.

O nível de atividade física (NAF) foi estimado utilizando-se o Self-Administered Activity Checklist (SALLIS et al., 1996), em sua versão adaptada e validada para a população brasileira (FARIAS JÚNIOR et al., 2012). Neste questionário os adolescentes reportaram a frequência semanal e a duração na participação de até 25 tipos de atividades físicas, com inten- sidades moderadas a vigorosas na última semana. $\mathrm{O}$ escore da atividade física foi calculado com a soma do produto da frequência semanal pelo volume, em minutos, despendido em cada atividade. Para as análises, foram considerados insuficientemente ativos os adolescentes com um volume semanal de atividades físicas menor que 420 minutos (WHO, 2010). O instrumento apresenta coeficiente de correlação intraclasse (CCI) de 0,88, uma correlação de Spearman de 0,62 $(p<0,001)$ e índice Kappa de 0,59 (FARIAS JÚNIOR et al., 2012).

O nível socioeconômico (NSE) foi avaliado com base no número de utensílios domésticos da residência do avaliado, na presença ou não de empregada mensalista e na escolaridade do responsável financeiro pelo domicílio (ABEP, 2013). Para as análises, os adolescentes foram classificados em três categorias: baixo NSE (classes C e D), NSE intermediário (classes B1 e B2) e elevado NSE (classes A1 e A2).

A maturação sexual foi determinada por meio do método proposto por Tanner (1962), no qual os estágios maturacionais dividem-se entre 1 (pré-púbere), 2, 3 e 4 (púbere) e 5 (pós-púbere). A classificação nos estágios foi autoavaliada pelos próprios adolescentes mediante a análise da pilosidade pubiana por intermédio de imagens (MARTIN et al., 2001; BOJIKIAN et al., 2002). Os adolescentes pré-púberes, entretanto, foram excluídos das análises do presente estudo por este estágio ser considerado uma fase de pré-adolescência (MALINA et al., 2015).

A massa corporal total foi mensurada com uma balança digital portátil da marca Plenna, com resolução de $100 \mathrm{~g}$, e a altura com um estadiômetro vertical portátil (Wiso) escalonado em 0,1 cm. Com estes dados foi possível calcular o índice de massa corporal a partir da razão entre a massa corporal e a estatura ao quadrado (Massa corpórea $(\mathrm{Kg}) / a$ ltura $\left.(\mathrm{m})^{2}\right)$. Posteriormente os adolescentes foram classificados de acordo com os critérios da Organização Mundial da Saúde (WHO, 2006) para o IMC em relação à idade a partir do cálculo do escore $Z$. Para fins de análise, os adolescentes foram divididos em "eutrófico" (baixo peso e peso normal) e "excesso de peso" (sobrepeso e obeso) (baseado nos pontos de corte de $\leq 1$ desvio padrão como eutrófico e acima de $>1$ desvio padrão como excesso de peso).

O tempo em diferentes tipos de atividades sedentárias foi estimado por meio do Adolescents Sedentary Activity Questionnaire (Asaq) (HARDY; BOOTH; OKELY, 2007) em sua versão válida para a população brasileira (GUIMARÃES et al., 2013). O instrumento avalia quanto os adolescentes dispendem em tempo 
de tela recreacional (tempo de tela), tempo sentado em sala de aula (educacional), tocando/praticando um instrumento musical (cultural), conversando com amigos ou relaxando (social) e se deslocando de carro/ônibus/moto (transporte). Nas análises, para todas as atividades foi considerado como elevado tempo sedentário uma quantidade de minutos acima do percentil 50 da distribuição da própria amostra. 0 instrumento apresenta um $\mathrm{CCl}=0,90$ com IC95\%: 0,86-0,93 (GUIMARÃES et al., 2013).

\section{Análise dos Dados}

Para o tratamento dos dados, inicialmente frequências absolutas e relativas foram utilizadas para descrever a amostra, quando as possíveis diferenças entre sexos foram verificadas pelo teste de qui-quadrado. As possíveis associações foram verificadas em dois diferentes modelos de regressão de Poisson com variância robusta. 0 modelo 1 reportou as análises brutas entre os fatores e os diferentes tipos de comportamento sedentário. No modelo 2, para as análi- ses ajustadas, foram inseridos apenas os fatores que apresentaram um valor de $p \leq 0,20$ no modelo 1 , para verificar quais fatores realmente se associam aos diferentes comportamentos sedentários (Tabela 3). Todas as análises foram realizadas no software SPSS 24.0, com um nível de confiança estabelecido em $95 \%$.

\section{RESULTADOS}

A amostra final foi composta por 785 adolescentes ( $53,7 \%$ do sexo feminino) com média de idade de 16,19 $\pm 1,11$ anos. Em relação às meninas, $60,2 \%$ e $55,9 \%$ apresentaram elevado tempo em atividades culturais e sociais $(p=0,01)$, enquanto para os meninos o comportamento mais frequente foi o tempo de tela com $54,8 \%(p=0,01)$. Sobre os adolescentes, de ambos os sexos, que não cumpriram as recomendações mínimas para atividades físicas, 49,9\% destes apresentaram um elevado tempo sedentário em relação ao seu deslocamento $(p=0,01)$. Adolescentes pós-púberes mostraram prevalências de $40,9 \%$ de elevado tempo em atividades sociais $(p=0,01)$ (Tabela 1 ).

Tabela 1 - Prevalência de tempo elevado nos diferentes tipos de comportamento sedentário analisados - São José dos Pinhais, Paraná, Brasil, $2014(n=785)$

\begin{tabular}{|c|c|c|c|c|c|c|c|c|c|c|c|c|c|c|c|}
\hline & \multicolumn{3}{|c|}{ Tela } & \multicolumn{3}{|c|}{ Educacional } & \multicolumn{3}{|c|}{ Cultural } & \multicolumn{3}{|c|}{ Social } & \multicolumn{3}{|c|}{ Transporte } \\
\hline & $\mathbf{n}$ & $\%$ & $p$ & $\mathbf{n}$ & $\%$ & $p$ & $\mathbf{n}$ & $\%$ & $p$ & $\mathbf{n}$ & $\%$ & $p$ & $\mathbf{n}$ & $\%$ & $p$ \\
\hline \multicolumn{16}{|l|}{ Sexo } \\
\hline Masculino & 199 & 54,8 & $0,01 *$ & 134 & 36,9 & $0,01^{*}$ & 69 & 19,0 & $0,01 *$ & 153 & 42,1 & $0,01 *$ & 179 & 49,3 & 0,80 \\
\hline Feminino & 191 & 45,3 & & 254 & 60,2 & & 123 & 29,1 & & 236 & 55,9 & & 213 & 50,5 & \\
\hline \multicolumn{16}{|c|}{ Nível de atividade física } \\
\hline Suficientemente ativo & 66 & 55,0 & 0,24 & 51 & 42,5 & 0,12 & 35 & 29,2 & 0,23 & 63 & 52,5 & 0,54 & 74 & 61,7 & $0,01^{*}$ \\
\hline Insuficientemente ativo & 324 & 48,7 & & 337 & 50,7 & & 157 & 23,6 & & 326 & 49,0 & & 318 & 47,8 & \\
\hline \multicolumn{16}{|l|}{ Nível socioeconômico } \\
\hline Elevado & 119 & 49,8 & 0,46 & 117 & 49,0 & 0,71 & 57 & 23,8 & 0,57 & 118 & 49,4 & 0,61 & 129 & 54,0 & 0,25 \\
\hline Intermediário & 230 & 51,1 & & 222 & 49,3 & & 109 & 24,2 & & 220 & 48,9 & & 216 & 48,0 & \\
\hline Baixo & 41 & 43,2 & & 49 & 51,6 & & 26 & 27,4 & & 51 & 53,7 & & 47 & 49,5 & \\
\hline \multicolumn{16}{|l|}{ Maturação sexual } \\
\hline Púbere & 270 & 50,2 & 0,73 & 272 & 50,6 & 0,39 & 142 & 26,4 & 0,07 & 288 & 53,5 & $0,01 *$ & 275 & 51,1 & 0,36 \\
\hline Pós-púbere & 120 & 48,6 & & 116 & 47,0 & & 50 & 20,2 & & 101 & 40,9 & & 117 & 47,4 & \\
\hline \multicolumn{16}{|l|}{ Estado nutricional } \\
\hline Sem excesso de peso & 370 & 49,7 & 1,00 & 365 & 49,1 & 0,47 & 178 & 23,9 & 0,19 & 370 & 49,7 & 0,79 & 374 & 50,3 & 0,52 \\
\hline Excesso de peso & 20 & 48,8 & & 23 & 56,1 & & 14 & 34,1 & & 19 & 46,3 & & 18 & 43,9 & \\
\hline
\end{tabular}

* Significativo para o teste de qui-quadrado de correção de continuidade; $p<0,05$. 
Tabela 2 - Associação do sexo, nível de atividade física, nível socioeconômico, maturação sexual e estado nutricional com os diferentes tipos de comportamento sedentário - São José dos Pinhais, Paraná, Brasil, 2014 ( $n=785)$

\begin{tabular}{|c|c|c|c|c|c|c|c|c|c|c|c|c|c|c|c|}
\hline \multirow[b]{3}{*}{ Análise bruta } & \multicolumn{3}{|c|}{ Tela } & \multicolumn{3}{|c|}{ Educacional } & \multicolumn{3}{|c|}{ Cultural } & \multicolumn{3}{|c|}{ Social } & \multicolumn{3}{|c|}{ Transporte } \\
\hline & RP & IC95\% & $p$ & RP & IC95\% & $p$ & $\mathrm{RP}$ & IC95\% & $\mathrm{p}$ & $\mathrm{RP}$ & IC95\% & $p$ & $\mathrm{RP}$ & IC95\% & $p$ \\
\hline & & & & & & & & & & & & & & & \\
\hline \multicolumn{16}{|l|}{ Sexo } \\
\hline Masculino & 1 & - & - & 1 & - & - & 1 & - & - & 1 & - & - & 1 & - & - \\
\hline Feminino & 0,82 & $0,71-0,95$ & 0,01 & 1,63 & $1,39-1,90$ & 0,01 & 1,53 & $1,18-1,99$ & 0,01 & 1,33 & $1,14-1,54$ & 0,01 & 1,02 & $0,89-1,18$ & 0,72 \\
\hline \multicolumn{16}{|l|}{$\begin{array}{l}\text { Nível de atividade } \\
\text { física }\end{array}$} \\
\hline $\begin{array}{l}\text { Suficientemente } \\
\text { ativo }\end{array}$ & 1 & - & - & 1 & - & - & 1 & - & - & 1 & - & - & 1 & - & - \\
\hline $\begin{array}{l}\text { Insuficientemente } \\
\text { ativo }\end{array}$ & 0,88 & $0,74-1,06$ & 0,19 & 1,19 & $0,95-1,49$ & 0,11 & 0,81 & $0,59-1,10$ & 0,18 & 0,93 & $0,77-1,12$ & 0,48 & 0,77 & $0,66-0,91$ & 0,01 \\
\hline \multicolumn{16}{|l|}{$\begin{array}{c}\text { Nível } \\
\text { socioeconômico }\end{array}$} \\
\hline Elevado & 1 & - & - & 1 & - & - & 1 & - & - & 1 & - & - & 1 & - & - \\
\hline Intermediário & 1,02 & $0,87-1,20$ & 0,74 & 1,00 & $0,85-1,18$ & 0,92 & 1,01 & $0,76-1,34$ & 0,91 & 0,99 & $0,84-1,16$ & 0,90 & 0,88 & $0,76-1,03$ & 0,12 \\
\hline Baixo & 0,86 & $0,66-1,12$ & 0,28 & 1,05 & $0,83-1,33$ & 0,66 & 1,14 & $0,77-1,70$ & 0,49 & 1,08 & $0,86-1,36$ & 0,46 & 0,91 & $0,72-1,15$ & 0,46 \\
\hline \multicolumn{16}{|l|}{ Maturação sexual } \\
\hline Púbere & 1 & - & - & 1 & - & - & 1 & - & - & 1 & - & - & 1 & - & - \\
\hline Pós-púbere & 0,97 & $0,83-1,13$ & 0,71 & 0,93 & $0,79-1,09$ & 0,38 & 0,77 & $0,57-1,02$ & 0,07 & 0,76 & $0,64-0,90$ & 0,01 & 0,93 & $0,79-1,08$ & 0,36 \\
\hline \multicolumn{16}{|l|}{$\begin{array}{c}\text { Estado } \\
\text { nutricional }\end{array}$} \\
\hline $\begin{array}{l}\text { Sem excesso de } \\
\text { peso }\end{array}$ & 1 & - & - & 1 & - & - & 1 & - & - & 1 & - & - & 1 & - & - \\
\hline Excesso de peso & 0,98 & $0,71-1,35$ & 0,90 & 1,14 & $0,86-1,51$ & 0,35 & 1,42 & $0,91-2,22$ & 0,11 & 0,93 & $0,66-1,30$ & 0,67 & 0,87 & $0,61-1,24$ & 0,44 \\
\hline
\end{tabular}

No modelo 1 foram observadas associações positivas entre o sexo feminino e o elevado tempo de tela (RP: 0,82; IC95\%: 0,71-0,95), em atividades educacionais (RP: 1,63; IC95\%: $1,39-1,90)$, culturais (RP: 1,53; IC95\%: $1,18-1,99$ ) e sociais (RP: 1,33 ; IC95\%: $1,14-1,54)$. Entre os adolescentes que não cumpriram as recomendações para atividade física, as associações observadas foram negativas com o transporte (RP: 0,77; IC95\%: 0,66-0,91). Para os adolescentes pós-púberes foram observadas associações com o tempo em atividades sociais (RP: 0,76; IC95\%: 0,64$0,90)$ (Tabela 2).
Tabela 3 - Caracterização dos modelos de análise

\begin{tabular}{|c|c|}
\hline Modelo 1 & Modelo 2 \\
\hline \multirow[t]{2}{*}{ Análise bruta } & $\begin{array}{l}\text { Ajustado aos fatores com } p \leq 0,20 \text { em } \\
\text { relação ao desfecho }\end{array}$ \\
\hline & $\begin{array}{l}\text { - Tempo de tela (Sexo e nível de } \\
\text { atividade física) } \\
\text { - Educacional (Sexo e nível de atividade } \\
\text { física) } \\
\text { - Cultural (Sexo, maturação sexual, } \\
\text { estado nutricional e nível de atividade } \\
\text { física) } \\
\text { - Social (Sexo e maturação sexual) } \\
\text { - Transporte (Nível de atividade física e } \\
\text { nível socioeconômico) }\end{array}$ \\
\hline
\end{tabular}


Tabela 4 - Associação hierárquica do sexo, nível de atividade física, nível socioeconômico, maturação sexual e estado nutricional com os diferentes tipos de comportamento sedentário - São José dos Pinhais, Paraná, Brasil, 2014 ( $n=785)$

\begin{tabular}{|c|c|c|c|c|c|c|c|c|c|c|c|c|c|c|c|}
\hline & \multicolumn{3}{|c|}{ Tela } & \multicolumn{3}{|c|}{ Educacional } & \multicolumn{3}{|c|}{ Cultural } & \multicolumn{3}{|c|}{ Social } & \multicolumn{3}{|c|}{ Transporte } \\
\hline & $\mathbf{R P}$ & IC95\% & $p$ & $\mathbf{R P}$ & IC95\% & $p$ & $\mathbf{R P}$ & IC95\% & $p$ & $\mathbf{R P}$ & IC95\% & $p$ & $\mathbf{R P}$ & IC95\% & $p$ \\
\hline \multicolumn{16}{|l|}{ Análise ajustada } \\
\hline \multicolumn{16}{|l|}{ Sexo } \\
\hline Masculino & 1 & - & - & 1 & - & - & 1 & - & - & 1 & - & - & - & - & - \\
\hline Feminino & 0,83 & $0,72-0,96$ & 0,01 & 1,62 & $1,38-1,90$ & 0,01 & 0,41 & $0,30-0,56$ & 0,01 & 0,58 & $0,53-0,64$ & 0,01 & - & - & - \\
\hline \multicolumn{16}{|l|}{$\begin{array}{l}\text { Nível de atividade } \\
\text { física }\end{array}$} \\
\hline $\begin{array}{l}\text { Suficientemente } \\
\text { ativo }\end{array}$ & 1 & - & - & 1 & - & - & 1 & - & - & - & - & - & 1 & - & - \\
\hline $\begin{array}{l}\text { Insuficientemente } \\
\text { ativo }\end{array}$ & 0,93 & $0,77-1,12$ & 0,45 & 1,04 & $0,84-1,28$ & 0,71 & 0,68 & $0,49-0,94$ & 0,02 & - & - & - & 0,51 & $0,45-0,58$ & 0,01 \\
\hline \multicolumn{16}{|l|}{$\begin{array}{c}\text { Nível } \\
\text { socioeconômico }\end{array}$} \\
\hline Elevado & - & - & - & - & - & - & - & - & - & - & - & - & 1 & - & - \\
\hline Intermediário & - & - & - & - & - & - & - & - & - & - & - & - & 0,89 & $0,76-1,03$ & 0,14 \\
\hline Baixo & - & - & - & - & - & - & - & - & - & - & - & - & 0,94 & $0,74-1,19$ & 0,62 \\
\hline \multicolumn{16}{|l|}{ Maturação sexual } \\
\hline Púbere & 1 & - & - & - & - & - & 1 & - & - & 1 & - & - & - & - & - \\
\hline Pós-púbere & 1,01 & $0,99-1,04$ & 0,16 & - & - & - & 0,83 & $0,62-1,11$ & 0,22 & 0,80 & $0,68-0,95$ & 0,01 & - & - & - \\
\hline \multicolumn{16}{|l|}{$\begin{array}{c}\text { Estado } \\
\text { nutricional }\end{array}$} \\
\hline $\begin{array}{l}\text { Sem excesso de } \\
\text { peso }\end{array}$ & - & - & - & - & - & - & 1 & - & - & - & - & - & - & - & - \\
\hline Excesso de peso & - & - & - & - & - & - & 1,62 & $1,04-2,52$ & 0,03 & - & - & - & - & - & - \\
\hline
\end{tabular}

RP: razão de prevalência; IC9\%: intervalo de confiança de $95 \%$; $p<0,05$.

No modelo 2, o sexo feminino apresentou associação positiva com o elevado tempo de tela (RP: 0,83; IC95\%: 0,72-0,96), em atividades educacionais (RP: 1,62; IC95\%: $1,38-1,90)$, culturais (RP: 0,41; IC95\%: $0,30-0,56$ ) e sociais (RP: 0,58; IC95\%: 0,53-0,64). O nível insuficiente de atividade física foi negativamente associado ao elevado tempo em atividades culturais (RP: 0,68; IC95\%: 0,49-0,94) e de transporte (RP: 0,51; IC95\%: 0,45-0,58). O estágio maturacional pós-púbere mostrou associação com o elevado tempo em atividades sociais (RP: 0,80; IC95\%: 0,68-0,95). Os adolescentes em excesso de peso apresentaram associação positiva com o elevado tempo em atividades culturais (RP: 1,62; IC95\%: 1,04-2,52) (Tabela 4).

\section{DISCUSSÃO}

Os achados do presente estudo indicaram que o sexo feminino, o nível de atividade física, a maturação sexual e o estado nutricional se associam de maneiras diferentes com o comportamento sedentário; resultados que contribuem para a literatura científica, uma vez que as evidências até então disponíveis se limitam a apresentar prevalências e associações destes fatores basicamente do tempo de tela (BARBOSA FILHO; CAMPOS; LOPES, 2014; GUERRA; FARIAS JÚNIOR; FLORINDO, 2016; KANTOMAA et al., 2016). Vale ressaltar, também, a análise hierárquica utilizada, o que 
permite verificar quais fatores realmente ajudariam a explicar os diferentes comportamentos sedentários investigados.

Meninos e meninas diferem em relação às preferências por atividades físicas, bem como seu nível (BARBOSA FILHO; CAMPOS; LOPES, 2014; COLL et al., 2014; DUMITH et al., 2016), tendência também observada no comportamento sedentário que, com a exceção do tempo em atividades educacionais, o sexo feminino apresenta-se como um fator de proteção aos outros comportamentos sedentários. A explicação talvez se deva por que culturalmente as meninas são mais incentivadas a atividades expressivas (SEABRA et al., 2008) e às transformações corporais, e, em proporções associadas à adolescência, podem dificultar o desempenho motor e fisiológico, favorecendo a diminuição das atividades físicas; com isso surgem mudanças nos padrões de comportamento, aumentando as obrigações com tarefas diárias, trabalhos domésticos e até a transição da escola para o trabalho (BACIL et al., 2016), fatos que podem contribuir para a diminuição da prática habitual de atividades físicas, mas, aparentemente, não para o aumento do comportamento sedentário.

Os resultados observados pelo presente estudo indicaram uma associação negativa entre o nível insuficiente de atividades físicas e o tempo em atividades culturais e deslocamento por transporte motorizado. Fatores socioculturais da atividade física costumam estar associados à influência de médicos, ao ambiente construído e pelo apoio social da família, amigos e professores (SEABRA et al., 2008; BAUMAN et al., 2012), o que poderia explicar a associação inversa entre o nível insuficiente de atividades físicas e as atividades culturais. A literatura também evidencia que um deslocamento ativo pode estar associado à segurança do bairro, qualidade de parques, infraestrutura para o deslocamento ativo (SMITH et al., 2017) e distância de casa para a escola (DUNCAN et al., 2016), o que nos leva a crer que a associação negativa não necessariamente representaria um maior deslocamento ativo, mas outros fatores, como a preferência por outras atividades, a preguiça, outras tarefas e até o transporte escolar oferecido pelo município (DIAS; LOCH; RONQUE, 2015).

Adolescentes mais maturados tendem a ter amigos mais velhos e igualmente mais maturados. Para estar em conformidade com as normas de certos grupos e ter participação e identidade, os adolescentes acabam por aderir a comportamentos semelhantes aos destes, pois, embora fisicamente desenvolvido, podem ser psicologicamente imaturos e sem habilidades cognitivas para resistir à pressão social de seus amigos, que tendem a realizar mais comportamentos sedentários (BACIL et al., 2016).

Sobre a associação inversa entre adolescentes maturados e o elevado tempo em atividades culturais, embora as evidências possam indicar que a maturação possa contribuir para um maior comportamento sedentário, essa relação pode variar (BACIL et al., 2015), além de haver resultados inconclusivos em razão de a literatura disponível analisar basicamente o tempo de tela como comportamento sedentário (BARBOSA FILHO et al., 2012; BARBOSA FILHO; CAMPOS; LOPES, 2014; FERREIRA et al., 2016; GUERRA; FARIAS JÚNIOR; FLORINDO, 2016).

É sabido que adolescentes com excesso de peso tendem a apresentar menores rendimentos acadêmicos, autoimagem negativa, depressão, exclusão social e maiores níveis de depressão (RIVERA et al., 2014; (NCD-RISC), 2017). Também é conhecido o fato de que o estado nutricional de adolescentes tende a se associar com outros fatores contrários à atividade física (BARBOSA FILHO et al., 2012; FERRARI et al., 2017), o que explicaria a preferência por atividades mais reclusas, como aprender a tocar algum instrumento musical ou a prática de outros hobbies manuais, caracterizados como atividades culturais.

A presente investigação não é livre de limitações, afinal a amostra foi composta apenas por estudantes de escolas públicas. O método utilizado para a coleta de dados (autorreportados), embora amplamente utilizado pela literatura (TENÓRIO et al., 2010; BARBOSA FILHO et al., 2012; FERREIRA et al., 2016), tende a superestimar e subestimar as respostas além da limitação quanto à sua acurácia. Por outro lado, o estudo apresenta amostra representativa de estudantes de escola pública com excelente poder de análise relacionado ao $n$, e uma análise hierárquica visando a ajustar o modelo a varáveis que realmente possam explicar as associações, além de analisar diferentes desfechos do comportamento sedentário nesta população. Mostra, também, a apresentação de um ponto de corte estabelecido no percentil 50 da amostra (mediana), pois o presente estudo analisou diferentes tipos de comportamento sedentário, o que impossibilitaria a referência de duas horas da Associação Americana de Pediatria (BAR-ON et al., 2001), posto que esta se limita apenas ao uso de TV e o presente estudo considerou também computadores e video games.

Como conclusão, diversos fatores parecem se associar a diferentes tipos de comportamento sedentário. Meninas podem ser mais propensas a atividades educacionais, enquanto meninos parecem preferir 
mais atividades em tela, culturais e sociais, e o baixo nível de atividades físicas parece se associar negativamente com atividades culturais e com o transporte sedentário. A maturação tende a exercer um fator protetivo para atividades sociais. Por fim, os adolescentes com excesso de peso parecem estar mais direcionados a atividades culturais. Recomenda-se novas investigações sobre fatores possivelmente associados a diferentes tipos de comportamento sedentário, e que as intervenções visando à diminuição do comportamento sedentário considerem este desfecho como multifatorial e mais amplo do que apenas o tempo de tela.

\section{REFERÊNCIAS}

(NCD-RISC), N. R. F. C. Worldwide trends in body-mass index, underweight, overweight, and obesity from 1975 to 2016: a pooled analysis of 2416 population-based measurement studies in 128.9 million children, adolescents, and adults. The Lancet, v. 390, n. 10.113, p. 2.627-2.642, 2017.

ABEP. Associação Brasileira de Empresas de Pesquisa. Critério de Classificação Econômica Brasil. São Paulo: ABEP, 2013.

AINSWORTH, B. E.; HASKELL, W. L.; WHITT, M. C.; IRWIN, M. L.; SWARTZ, A. M.; STRATH, S. J.; O'BRIEN, W. L.; BASSETT, D. R. JR.; SCHMITZ, K. H.; EMPLAINCOURT, P. O.; JACOBS, D. R. JR.; LEON, A. S. Compendium of physical activities: an update of activity codes and MET intensities. Medicine \& Science in Sports \& Exercise, v. 32, n. 9 Suppl, p. S498-504, 2000.

BACIL, E. D.; MAZZARDO JUNIOR, O.; RECH, C. R.; LEGNANI, R. F.; CAMPOS, W. Physical activity and biological maturation: a systematic review. Revista Paulista de Pediatria, v. 33, n. 1, p. 114-21, 2015.

BACIL, E. D. A.; PIOLA, T. S.; WATANABE, P. I.; SILVA, M. P.; LEGNANI, R. F. S.; CAMPOS, W. Biological maturation and sedentary behavior in children and adolescents: a systematic review. Journal of Physical Education, v. 27, p. e27302016, 2016.

BAR-ON, Miriam E.; BROUGHTON, Daniel D.; BUTTROSS, Susan; CORRIGAN, Suzanne; GEDISSMAN, Alberto M.; GONZÁLEZ DE RIVAS, Rosario; RICH, Michael; SHIFRIN, Donald L.; BRODY, Michael; WILCOX, Brian L.; HOGAN, Marjorie H.; HOLROYD, James; REID, Linda S.; SHERRY, Norman; STRASBURGER, Victor; STONE, Jennifer. Children, adolescents, and television. Pediatrics, v. 107, n. 2, p. 423-426, 2001.

BARBOSA FILHO, V. C.; CAMPOS, W.; BOZZA, R.; LOPES, A. S. The prevalence and correlates of behavioral risk factors for cardiovascular health among Southern Brazil adolescents: a cross-sectional study. BMC Pediatrics, v. 12 , n. 130 , p. 12 , 2012.

BARBOSA FILHO, V. C.; CAMPOS, W.; LOPES, A. S. Epidemiology of physical inactivity, sedentary behaviors, and unhealthy eating habits among Brazilian adolescents: a systematic review. Ciência e Saúde Coletiva, v. 19, n. 1, p. 173-93, 2014.
BAUMAN, A. E.; REIS, R. S.; SALLIS, J. F.; WELLS, J. C.; LOOS, R. J.; MARTIN, B. W. Correlates of physical activity: why are some people physically active and others not? The Lancet, v. 380, n. 9.838, p. 258-271, 2012.

BOJIKIAN, L. P.; MASSA, M.; MARTIN, R. H. C.; TEIXEIRA, C. P.; KISS, M. A. P. D. M.; BÖHME, M. T. S. Auto-avaliação puberal feminina por meio de desenhos e fotos. Revista Brasileira de Atividade Física \& Saúde, v. 7, n. 2, p. 24-34, 2002.

BRASIL, A. Atlas do desenvolvimento humano no Brasil 2013. Programa das Nações Unidas para o Desenvolvimento - PNUD, 2016.

COLL, C. D. V. N.; KNUTH, A. G.; BASTOS, J. P.; HALLAL, P. C.; BERTOLDI, A. D. Time trends of physical activity among Brazilian adolescents over a 7-year period. Journal of Adolescent Health, v. 54, n. 2, p. 209-213, 2014.

DEMIDENKO, E. Poisson regression for clustered data. International Statistical Review, v. 75, n. 1, p. 96-113, 2007.

DIAS, D. F.; LOCH, M. R.; RONQUE, E. R. V. Barreiras percebidas à prática de atividades físicas no lazer e fatores associados em adolescentes. Ciência e Saúde Coletiva, v. 20, p. 3.339-3.350, 2015.

DIAS, P. J.; DOMINGOS, I. P.; FERREIRA, M. G.; MURARO, A. P.; SICHIERI, R.; GONCALVES-SILVA, R. M. Prevalence and factors associated with sedentary behavior in adolescents. Revista de Saúde Pública, v. 48, n. 2, p. 266-274, 2014.

DUMITH, S. C.; SANTOS, M. N. D.; TEIXEIRA, L. O.; CAZEIRO, C. C.; MAZZA, S. E. I.; CESAR, J. A. Prática de atividade física entre jovens em município do semiárido no Brasil. Ciência e Saúde Coletiva, v. 21, p. 1.083-1.093, 2016.

DUNCAN, S.; WHITE, K.; MAVOA, S.; STEWART, T.; HINCKSON, E.; SCHOFIELD, G. Active transport, physical activity, and distance between home and school in children and adolescents. International Journal of Physical Activity and Health, v. 13, n. 4, p. 447-453, 2016.

EKELUND, U.; STEENE-JOHANNESSEN, J.; BROWN, W. J.; FAGERLAND, M. W.; OWEN, N.; POWELL, K. E.; BAUMAN, A.; LEE, I. M. Does physical activity attenuate, or even eliminate, the detrimental association of sitting time with mortality? A harmonised meta-analysis of data from more than 1 million men and women. The Lancet, v. 388, n. 10.051, p. 1.302-1.310, 2016.

FARIAS JÚNIOR, J. C.; LOPES, A. S.; MOTA, J.; SANTOS, M. P.; RIBEIRO, J. C.; HALLAL, P. C. Validade e reprodutibilidade de um questionário para medida de atividade física em adolescentes: uma adaptação do Self-Administered Physical Activity Checklist. Revista Brasileira de Epidemiologia, v. 15, n. 1, p. 198-210, 2012.

FERRARI, G. L. D. M.; MATSUDO, V.; KATZMARZYK, P. T.; FISBERG, M. Prevalence and factors associated with body mass index in children aged 9-11 years. Jornal de Pediatria, v. 93, p. 601-609, 2017.

FERREIRA, R. W.; ROMBALDI, A. J.; RICARDO, L. I. C.; HALLAL, P. C.; AZEVEDO, M. R. Prevalence of sedentary behavior and its correlates among primary and secondary school students. Revista Paulista de Pediatria, v. 34, n. 1, p. 56-63, 2016. 
GUERRA, P. H.; FARIAS JÚNIOR, J. C.; FLORINDO, A. A. Comportamento sedentário em crianças e adolescentes brasileiros: revisão sistemática. Revista de Saúde Pública, v. 50, n. 9, p. 2-15, 2016.

GUIMARÃES, R. F.; SILVA, M. P.; LEGNANI, E.; MAZZARDO, O.; CAMPOS, W. Reproducibility of adolescent sedentary activity questionnaire (ASAQ) in Brazilian adolescents. Revista Brasileira de Cineantropometria e Desempenho Humano, v. 15, n. 3, p. 276-285, 2013.

HALLAL, P. C.; ANDERSEN, L. B.; BULL, F. C.; GUTHOLD, R.; HASKELL, W.; EKELUND, U. Global physical activity levels: surveillance progress, pitfalls, and prospects. The Lancet, $\mathrm{v}$. 380, n. 9.838, p. 247-57, 2012.

HARDY, L. L.; BOOTH, M. L.; OKELY, A. D. The reliability of the Adolescent Sedentary Activity Questionnaire (ASAQ). Preventive Medicine, v. 45, n. 1, p. 71-74, 2007.

KANTOMAA, M. T.; STAMATAKIS, E.; KANKAANPÄÄ, A.; KAJANTIE, E.; TAANILA, A.; TAMMELIN, T. Associations of physical activity and sedentary behavior with adolescent academic achievement. Journal o Research on Adolescence, v. 26, n. 3, p. 432-442, 2016.

LUIZ, R. R.; MAGNANINI, M. M. F. A lógica da determinação do tamanho da amostra em investigações epidemiológicas. Cadernos de Saúde Coletiva, v. 8, n. 2, p. 9-28, 2000.

MALINA, R. M.; ROGOL, A. D.; CUMMING, S. P.; SILVA, M. J. C.; FIGUEIREDO, A. J. Biological maturation of youth athletes: assessment and implications. British Journal of Sports Medicine, v. 49, n. 13, p. 852-859, 2015.

MARTIN, R. H. C.; UEZU, R.; PARRA, A.; ARENA, S.; BOJIKIAN, L.; BOHME, M. Auto-avaliação da maturação sexual masculina por meio da utilização de desenhos e fotos. Revista Brasileira de Educação Física e Esporte, v. 15, n. 2, p. 212-222, 2001.

PATE, R. R.; O'NEILL, J. R.; LOBELO, F. The evolving definition of "sedentary". Exercise and sport sciences reviews, v. 36, n. 4, p. 173-8, 2008.

RIVERA, J. Á.; DE COSSÍO, T. G.; PEDRAZA, L. S.; ABURTO, T. C.; SÁNCHEZ, T. G.; MARTORELL, R. Childhood and adolescent overweight and obesity in Latin America: a systematic review. The Lancet, v. 2, n. 4, p. 321-332, 2014.
SALLIS, J. F.; STRIKMILLER, P. K.; HARSHA, D. W.; FELDMAN, H. A.; EHLINGER, S.; STONE, E. J.; WILLISTON, J.; WOODS, S. Validation of interviewer- and self-administered physical activity checklists for fifth grade students. Medicine \& Science in Sports \& Exercise, v. 28, n. 7, p. 840-851, 1996.

SEABRA, A. F.; MENDONCA, D. M.; THOMIS, M. A.; ANJOS, L. A.; MAIA, J. A. Biological and socio-cultural determinants of physical activity in adolescents. Cadernos de Saúde Pública, v. 24, n. 4, p. 721-736, 2008.

SEED-PR. Consulta Escolas. 2014. Disponível em: http:// www.consultaescolas.pr.gov.br/consultaescolas/f/fcls/municipio/consultasEnsino.xhtml?cid=1. Acesso em: fev. 2014.

SMITH, M.; HOSKING, J.; WOODWARD, A.; WITTEN, K.; MACMILLAN, A.; FIELD, A.; BAAS, P.; MACKIE, H. Systematic literature review of built environment effects on physical activity and active transport - an update and new findings on health equity International. Journal of Behavioral Nutrition and Physical Activit, v. 14, n. 1, p. 158, 2017.

TANNER, J. M. (ed.). Growth at adolescence. Philadelphia (EUA): J. B. Lippincott Company, 1962.

TENÓRIO, M. C. M.; BARROS, M. V. G.; TASSITANO, R. M.; BEZERRA, J.; TENÓRIO, J. M.; HALLAL, P. C. Atividade física e comportamento sedentário em adolescentes estudantes do Ensino Médio. Revista Brasileira de Epidemiologia, v. 13, p. 105-117, 2010.

WHO. World Health Organization. WHO child growth standards: length/height for age, weight-for-age, weight-for-length, weight-for-height and body mass index-for-age, methods and development. Geneva: WHO, 2006.

WHO. World Health Organization. Global recommendations on physical activity for health. Geneva: WHO, 2010.

WHO. World Health Organization. Global accelerated action for the health of adolescents (AA-HA!): guidance to support country implementation. Geneva: WHO, 2017. 